\title{
Memory function for a fluid of molecules interacting through steeply repulsive potentials
}

\author{
G. Rickayzen* and D. M. Heyes ${ }^{\dagger}$ \\ Division of Chemistry, School of Biomedical and Molecular Sciences, University of Surrey, Guildford GU2 7XH, United Kingdom
}

(Received 11 February 2005; published 30 June 2005)

\begin{abstract}
Previous studies of the properties of fluids of molecules interacting through steeply repulsive central potentials are extended to the investigation of the memory function. It is assumed that collisions are dominated by binary collisions and a general formula previously derived by Miyazaki, Srinivas, and Bagchi [J. Chem. Phys. 114, 6276 (2001)] is applied to the present problem. It is shown that the equations of motion of a pair of molecules can be solved explicitly and substitution of the result into the formula leads to a closed explicit expression for the memory function which is easily evaluated for any given state. In the limit of hard spheres this result leads to Enskog's equation and represents a generalization of that formula to fluids with softer potentials. The results obtained from the formula are compared with those derived from the molecular dynamics simulation. The velocity autocorrelation function was calculated using the generalized soft sphere potential, $\phi(r)=\varepsilon(\sigma / r)^{n}$, where $\varepsilon$ and $\sigma$ set the energy and size of the molecule, and the exponent, $n$, is a variable. The two approaches agree very well for a range of state points for $n$ large, especially at short times.
\end{abstract}

DOI: 10.1103/PhysRevE.71.061204 PACS number(s): 66.10.Cb, 51.10.+y, 52.25.Fi, 52.65.Yy

\section{INTRODUCTION}

A longstanding goal in the theory of liquids has been that of predicting the molecular dynamics and transport properties for a given intermolecular potential, based solely on first principle statistical mechanical arguments. The first step in this direction was made by Enskog, who developed a kinetic theory of the transport coefficients of hard sphere fluids based on the Boltzmann equation (see, e.g., Ref. [1] for a good account). The assumptions in his theory are that each collision is instantaneous (i.e., a delta function in time) and independent of other previous collisions (i.e., it ignores correlated collision sequences), and the collision rate is proportional to the local density around a given particle (measured through the contact value of the radial distribution function). Actually the formulas he derived for the transport coefficients are surprisingly accurate up to quite high (liquidlike) densities, especially for the thermal conductivity [2]. Empirical correction formulas to the basic Enskog expressions based on Molecular Dynamics computer simulation data have been derived for the four transport coefficients (see for example, Ref. [2]). The hard-sphere fluid is, however, somewhat pathological as far as the particle dynamics is concerned [3] and of course such a potential does not exist in nature.

There has been some success in extending Enskog's original treatment to arbitrary continuous potentials (see Refs. [1,4], Secs. 2.7-2.9, for discussion of this), but an alternative approach based on the work of Green [5] and Kubo [6] has attracted more attention in recent years. This "Green-Kubo" treatment expresses a transport coefficient as (apart from a system constant) the integral of a time autocorrelation function, $\psi(t)=\langle A(0) A(t)\rangle$, where $t$ is time and $\langle\cdots\rangle$ denotes an average over time origins. The quantity $A$ is a flux which can

\footnotetext{
*Electronic address: g.rickayzen@surrey.ac.uk

†Electronic address: d.heyes@surrey.ac.uk
}

be defined at the molecular level in terms of various quantities including the intermolecular potential. Therefore knowledge of the time correlation function leads automatically to the transport coefficient. Although the interaction potential and the relevant time correlation are implicitly linked, to date closed analytic form solutions for arbitrary realistic potentials valid at all times, have eluded us. Nevertheless, the formally exact nature of the time correlation function approach does provide a sound basis for developing approximate solutions $\psi(t)$ and, hence, the transport coefficient. For example, using projection operators for relevant and weakly coupled degrees of freedom, it can be shown that the time correlation function can be expressed in terms of a more fundamental time dependent ("memory") function, $\zeta(t)$, in the form of an integrodifferential equation called the generalized Langevin equation (GLE) (see e.g., Chap. 9 in Ref. [7] and Sec. 2.6 in Ref. [4]). Indeed the memory function can itself be expressed as a GLE, and so on as a hierarchy. A closed expression for $\psi$ requires the inclusion of a specific functional form for the memory function at some termination stage. Normally the Laplace transform (LT) of these equations is considered, which forms a continued fraction expansion to be closed at $n$th order by the LT of such a terminating function. Alternatively the series can be continued indefinitely by assuming a relationship between the memory functions at arbitrary levels $n$ and $n+1$ (see for example Refs. $[8,9])$. An alternative approach is to attempt to derive an expression for the memory function of a particular fluid from first principles [10], and this is the approach taken in this work.

The particular fluid we study is one consisting of "molecules" interacting through a steeply repulsive central potential. In previous publications [11], we have studied several thermodynamic properties of such fluids which approach those of the hard sphere fluid as the steepness rises. We find small quantitative corrections to the hard sphere results. For the time relaxation of various static properties and fluxes we expect a more dramatic change as the hard sphere fluid dis- 
plays singular properties for small times (see Sec. 7.2 of Ref. [7]). Indeed the memory function of the velocity autocorrelation function (VACF) of the hard sphere fluid contains a delta function for short times (see Sec. 3.3 of Ref. [4]) whereas for continuous potentials it is analytic. We expect to see this difference in our study.

We concentrate on the VACF, $\psi(t)=\left\langle v_{i}(0) v_{i}(t)\right\rangle$, which, via the Green-Kubo formula, gives the self-diffusion coefficient

$$
D=(1 / 3) \int_{0}^{\infty} \psi(t) d t .
$$

Our approach is based on use of Eq. (16) in [K. Miyazaki, G. Srinivas, and B. Bagchi, J. Chem. Phys. 114, 6276 (2001)] (referred to as MSB) given in this paper as Eq. (2.2), which gives an explicit real time expression for the memory function in terms of the radial distribution function, $g(r)$, the Liouville, operator, and the pair potential. We find an approximate analytic solution for short times for steeply repulsive potentials, in which there is a clear separation of time scales between the binary collision itself and subsequent collisions. By solving the equations of relative motion of a pair of particles for any initial condition, we have obtained an explicit expression for the integrand of that expression, and hence, the memory function. As we ignore multiparticle scattering, mode coupling, and hydrodynamic effects, we expect that our results will be accurate only for short times.

In a recent paper Dufty and Ernst [3] have reported a calculation of the velocity and force autocorrelation functions for a steeply repulsive potential. They also include only the effect of binary collisions and express their results for short times in terms of a scaling function. As their physical assumptions are the same as ours, there should be a relationship between their results for the correlation functions and our results for the memory function. As they have not yet published the full details of their calculation we have not yet been able to establish this relationship although both produce numerical results which agree with computer simulation. We discuss this further in our concluding section.

\section{THE MEMORY FUNCTION FOR STEEPLY REPULSIVE POTENTIALS}

When two hard spheres collide each moves freely until the moment of collision at which time the separation of their centers is the molecular diameter, $\sigma$. After the collision the spheres again move freely but with a momentum altered by the collision. The collision takes place instantaneously and at the separation, $\sigma$. As was pointed out in Sec. I, one consequence of this is that at short times the memory function for a hard sphere fluid is abnormal. In fact it contains a delta function in $t$ whereas the expansion is normally analytic in $t$ (see Ref. [12]).

Similarly when two molecules moving with thermal speeds interact through a steeply repulsive potential, they will influence each other only within a short distance of an appropriate hard sphere separation, $\sigma$. The collision will take place only over a short time and only when the separation is close to $\sigma$. They will start to influence each other significantly only when their separation is slightly greater than $\sigma$ and they will penetrate each other slightly, i.e., their minimum separation will be slightly less than $\sigma$. The collision, therefore, takes place over a short but finite time and within a short part of their separation close to $\sigma$. These facts enable us to solve the equations of motion with a consistent approximation. Since the collision takes a finite, even if short, time the expansion of the autocorrelation functions is now normal and quadratic in $t$.

As stated in the introduction our approach to this problem is through the memory function, $\zeta(t)$, for a fluid. Implicitly the memory function is related to the velocity autocorrelation function, $\psi(t)$. In terms of their respective Laplace transforms, $\widetilde{\zeta}(z)$ and $\tilde{\psi}(z)$, the relationship is

$$
[m z+\widetilde{\zeta}(z)] \widetilde{\psi}(z)=\psi(0),
$$

where $m$ is the mass of a fluid molecule. This is simply a generalization of the Langevin equation. A formal definition of the memory function has been given by Zwanzig [13] and by Mori [14] but we do not need to use it here. Instead we use a result for the memory function derived by Miyazaki, Srivivas, and Bagchi [10]. Starting from the formal definition of the memory function and using results due to Mazenko and Yip [15] they derive, for a one-component fluid of molecules interacting through two-body interactions only and with the assumption that the transport properties are dominated by binary collisions, a closed expression for the memory function. For molecules interacting through a central intermolecular potential, $\phi(r)$, their result can be expressed in terms of the pair distribution function of the fluid, $g(r)$, and of the Boltzmann distribution function for a particle of mass $m / 2$; their formula for the memory function in this case is

$$
\zeta(t)=-\frac{\rho}{3} \sum_{\alpha=1 \ldots 3} \int d^{3} p \int d^{3} r f(p) \frac{\partial g(r)}{\partial r_{\alpha}} \frac{\partial \phi(\mathbf{r}(t))}{\partial r_{\alpha}(t)},
$$

where

$$
f(p)=\left(\pi m k_{B} T\right)^{-3 / 2} \exp \left(-p^{2} / m k_{B} T\right) .
$$

The vector $\boldsymbol{r}(t)$ is the radius vector between the centers of a pair of molecules at a time $t$ when their separation and relative momentum at the initial time were $\boldsymbol{r}$ and $\boldsymbol{p}$; it describes their relative motion in their center-of-mass frame.

In this section we evaluate the memory function, as far as is possible analytically, for the case of a fluid of molecules interacting through the steeply repulsive model central potential

$$
\phi(r)=\varepsilon\left(\frac{\sigma}{r}\right)^{n},
$$

in the limit that $\mathrm{n}$ becomes very large. When $n \rightarrow \infty$ the potential becomes the hard sphere one. In the hard-sphere limit the collision is instantaneous and the memory function is local and as already pointed out becomes a delta function [16]. But when $n$ is finite the collision does take time, even if 
it is a short time, and we wish to see what difference this makes to the memory function. Although we use the specific potential as given by Eq. (2.4) in this section, it is shown in Sec. IV that the theory applies mutatis mutandis to other steeply repulsive potentials (SRPs).

From thermodynamics [17] and from irreversible processes [18] it has been shown that for SRPs the optimal choice for the diameter of the hard sphere reference fluid is that given by the Barker-Henderson equation [17]. For the particular potential defined by Eq. (2.4), to leading order in $1 / n$, this diameter is the $\sigma$ in the definition of the potential.

In order to evaluate the memory function from Eq. (2.2) we need to find $\phi(\mathbf{r}(t))$ by solving the equation of motion in the center-of-mass frame of the two molecules. We provide details of the calculation in the Appendix. The result is

$$
\frac{\phi(r(t))}{W}=\frac{4 \exp \left\{-\gamma\left[t+t_{m} \operatorname{sgn}\left(\cos \theta_{0}\right)\right]\right\}}{\left(1+\exp \left\{-\gamma\left[t+t_{m} \operatorname{sgn}\left(\cos \theta_{0}\right)\right]\right\}\right)^{2}},
$$

where $t_{m}$ is the time of closest approach of the molecules and is defined explicitly in the Appendix by Eq. (A7).

In polar coordinates the memory function can be written as

$$
\begin{aligned}
\zeta(t)= & -\frac{8 \pi^{2} \rho}{3} \\
& \times \int_{0}^{\infty} p^{2} d p \int_{0}^{\infty} r^{2} d r \int_{0}^{\pi} \sin \theta d \theta f(p) \frac{\partial g(r)}{\partial r_{\alpha}} \frac{\partial \phi(r(t))}{\partial r_{\alpha}(t)} .
\end{aligned}
$$

If we now retain only terms of leading order in $1 / n$, we can use the approximation

$$
\frac{\partial g[r]}{\partial r_{\alpha}} \frac{\partial \phi(r(t))}{\partial r_{\alpha}(t)}=\beta \frac{n}{\sigma}\{y[\sigma] \exp [-\beta \phi(r)]\} \frac{\partial \phi(r)}{\partial r} \phi[r(t)] .
$$

This expression must be substituted into Eq. (2.6) and the integrals performed. Because the function in Eq. (2.7) is very small except when $r \sim \sigma$ one can replace $r$ in the integrand by $\sigma$ except within the rapidly varying potential $\phi(r)$. The derivative $\partial \phi / \partial r$ can be exploited to change the variable of integration from $r$ to $\phi(r)$. Also one can change variable from $\theta$ to $\mu=\cos \theta$. As a result one finds that

$$
\begin{aligned}
\zeta(t)= & \frac{8 \pi^{2} n \rho \beta \sigma}{3} y[\sigma] \int_{0}^{\infty} p^{2} d p \int_{0}^{\infty} d \phi \int_{-1}^{1} d \mu f(p) \\
& \times \exp [-\beta \phi(r)] \phi[R]
\end{aligned}
$$

with $\phi(R) \equiv \phi(r(t))$ given by Eq. (2.5).

It is illuminating to replace the variables by dimensionless ones by substituting

$$
\begin{gathered}
p=\left(m k_{B} T\right)^{1 / 2} P, \\
\phi=k_{B} T \chi, \\
\phi[R]=k_{B} T \chi[R],
\end{gathered}
$$

and

$$
w=W / k_{B} T=P^{2} \mu^{2}+\chi .
$$

Then

$$
\exp \left(-\gamma t_{m}\right)=\frac{\sqrt{w}-P|\mu|}{\sqrt{w}+P|\mu|}
$$

and

$$
\chi[R]=\frac{4 w A}{(1+A)^{2}},
$$

where

$$
A=\exp \left\{-\gamma\left[t+\operatorname{sgn}(\mu) t_{m}\right]\right\}
$$

Also

$$
\gamma=2 n\left[\frac{k_{B} T}{m \sigma^{2}} w\right]^{1 / 2} .
$$

The final result is

$$
\zeta(t)=c K(x)
$$

where

$$
\begin{gathered}
c=n 4 \sqrt{\pi} \rho \sigma y[\sigma] k_{B} T=n\left(m k_{B} T\right)^{1 / 2} / \sigma \tau_{E}, \\
x=2 n\left(\frac{k_{B} T}{m \sigma^{2}}\right)^{1 / 2} t=\gamma_{A} t, \quad \text { say, } \\
K(x)=\frac{2}{3} \int_{0}^{\infty} P^{2} d P \int_{0}^{\infty} d \chi \int_{-1}^{1} d \mu \chi[R] \exp \left(-P^{2}-\chi\right),
\end{gathered}
$$

and $\tau_{E}$ in Eq. (2.18) is Enskog's collision time for hard spheres of diameter $\sigma$ (see Ref. [19]). Hence, $\zeta(t)$ is proportional to the single function $K(x)$. Earlier in this section we interpreted $\gamma^{-1}$ as the duration of an individual collision. Likewise, we interpret $\gamma_{A}^{-1}$ as the average duration of a collision. Hence, the variable $x$ is the time measured in units of the average duration of a collision.

In the leading approximation in $n$ used in this paper, $\zeta(t)$ does not depend upon $k_{B} T / \varepsilon$. Because $\chi[R]$ is the value of the repulsive potential at the time $t$, it is positive. Hence, in this approximation the memory function is positive at all times. Furthermore, as is required for a continuous potential it is analytic at short times.

\section{HARD SPHERES IN THE LIMIT $n \rightarrow \infty$}

Miyazaki et al. [10] have already shown that $\widetilde{\zeta}(0)$ and, hence, the diffusion constant agree with Enskog's result [12] in the hard sphere limit of $n \rightarrow \infty$. We wish to show that this is true for all values of $t$ despite the further approximations we have made.

To demonstrate this we need to make the dependence on $n$ explicit. Inspection of Eqs. (2.17)-(2.20) reveals that $\zeta(t)$ depends on $n$ through factors of $n$ in the overall constant $c$ and in $x$ but note that $x_{m} \equiv \gamma t_{m}$ is defined by Eq. (2.13) and is 
independent of $n$. To show the dependence on $n$ explicitly we write

$$
x=n t_{R} ; \quad \alpha=\exp \left(-\gamma t_{m}\right)=\exp \left(-x_{m}\right),
$$

and consider the limit

$$
\lim _{n \rightarrow \infty}(n \chi\{[R]\}) .
$$

When $t_{R} \neq 0$ this is

$$
\lim _{n \rightarrow \infty}\left(4 n w \frac{\exp \left[-n t_{R}+\operatorname{sgn}(\mu)\right]}{\left\{1+\exp \left[-n t_{R}+\operatorname{sgn}(\mu)\right]\right\}^{2}}\right)=0 .
$$

However, when $t_{R}=0$ the limit is infinite. Further, when $\mu<0$

$$
\int_{0}^{\infty} d y n \chi[R]=4 w n \int_{0}^{\infty} d y \frac{\alpha e^{-n y}}{\left(1+\alpha e^{-n y}\right)^{2}}=\frac{4 w}{1+\alpha} .
$$

Hence, when $\mu<0$

$$
\lim _{n \rightarrow \infty}(n \chi[R])=\frac{4 w}{1+\alpha} \delta\left(t_{R}\right) .
$$

When $\mu>0, \alpha$ is replaced by $\alpha^{-1}$ and the resulting limit is

$$
\frac{4 w \alpha}{1+\alpha} \delta\left(t_{R}\right)
$$

Now in the integral for $K(x)$ in Eq. (2.20) the contributions from negative and positive values of $\mu$ can be added together. The resulting integrations become elementary and yield

$$
\zeta(t)=\frac{2 m}{3 \tau_{E}} \delta(t) .
$$

When used in Eq. (2.1) this leads to the usual result for the vacf for hard spheres [12]

$$
\psi(t)=\psi(0) \exp \left(-\frac{2 t}{3 \tau_{E}}\right) .
$$

\section{OTHER STEEPLY REPULSIVE POTENTIALS}

Other steeply repulsive potentials can be treated in a similar way. The hard-sphere radius is defined as before by the Barker-Henderson formula. The specific potential enters the calculation at the derivation of the relationship between $\dot{R}$ and $\dot{\phi}$. In general

$$
\dot{\phi}(R)=\phi^{\prime}(R) \dot{R}=k(R) \phi(R) \dot{R},
$$

where

$$
k(R)=\frac{\phi^{\prime}(R)}{\phi(R)}=\frac{d \ln [\phi(R)]}{d r} .
$$

The advantage of introducing the function $k(R)$ is that, in the region where the potential is a rapidly varying function of position, this function is slowly varying. Our result is limited to steeply repulsive potentials for which the function $k(R)$ is slowly varying. In this general case the equation for the conservation of energy, Eq. (A4), becomes

$$
\frac{\dot{\phi}^{2}}{k^{2}(R) \phi^{2}(R)}=\frac{4 E}{m}-\frac{4 l^{2}}{m^{2} R^{2}}-\frac{4 \phi}{m} .
$$

Now, since one is particularly concerned to solve this equation in the neighborhood of $R=\sigma$ where only the potential is varying rapidly, one can, to leading order, replace $R$ and $k(R)$ by $\sigma$ and $k(\sigma)$ respectively, except within the potential and its derivatives, Eq. (4.3) then becomes

$$
\frac{\dot{\phi}^{2}}{k^{2}(\sigma) \phi^{2}(R)}=\frac{4 E}{m}-\frac{4 l^{2}}{m^{2} \sigma^{2}}-\frac{4 \phi}{m} .
$$

A comparison of this equation with Eq. (A4) shows that the effect of using different SRPs is to replace the steepness parameter $n$ by the steepness parameter $\sigma k(\sigma)$. The final result for the memory function is therefore unchanged except that the appropriate steepness parameter replaces $n$. As an example consider the repulsive exponential potential

$$
\phi(r)=\exp [\kappa(\sigma-r)] .
$$

for $\kappa \sigma$ very large. In this case

$$
k(R)=\kappa,
$$

and is actually constant. The steepness parameter is then $\kappa \sigma$ and this replaces $n$ in the formulas given in Eqs. (2.17)-(2.20) for the memory function.

\section{RESULTS AND DISCUSSION}

In order to test the approximations made in the previous sections we have compared results for the memory function computed from Eq. (2.17) to Eq. (2.20) with results from Molecular Dynamics simulation of the same fluid at the same state point. The memory function was computed by numerical integration of the integrodifferential Eq. (3.2.7) given on page 109 in Ref. [4].

$$
\zeta(t)=C_{F}(t)-\int_{0}^{t} d t^{\prime} \zeta\left(t-t^{\prime}\right) \dot{\psi}\left(t^{\prime}\right),
$$

where $C_{F}(t)$ is the force autocorrelation function (FACF) and $\zeta(0)=C_{F}(0)$. Both $C_{F}(t)$ and the velocity autocorrelation function, $\psi(t)$ are computed directly in the Molecular Dynamics simulation. These results are likely to be more reliable at short times rather than long ones.

We used units in which $m=\sigma=1$ and selected states for which $k_{B} T=1$. The effect of this is that $\zeta(t)$ is measured in units of $k_{B} T / \sigma^{2}$ and time is measured in units of $\sqrt{\left(m \sigma^{2} / k_{B} T\right)}$. We report the results for five different samples labeled A-E and with values of the parameters $n$ and the packing fraction, $\eta$ as given in Table I. For further details of the Molecular Dynamics simulations see for example Ref. [11]. In Figs. 1-3 we compare the results of the theory with those calculated from simulation. In all the figures the subscripts A-E refer to the different simulations while the sub- 
TABLE I. The values of the parameters used in the various simulations. The parameter $n$ is the index occurring in the potential and packing fraction. $\eta=\pi \rho \sigma^{3} / 6$ is the packing fraction. $n$ is the exponent for the potential defined in Eq. (2.8). The simulations used $N=500$ particles and were carried out at a temperature $k_{B} T / \varepsilon=1$.

\begin{tabular}{ccc}
\hline \hline MD system & $n$ & $\eta$ \\
\hline A & 72 & 0.45 \\
B & 72 & 0.35 \\
C & 72 & 0.29 \\
D & 144 & 0.44 \\
E & 144 & 0.30 \\
\hline
\end{tabular}

script $T$ refers to the theory. The theory depends upon the cavity function and for that we use the Carnahan-Starling formula [20].

In Fig. 1 we compare the raw memory function as derived from simulation A plotted as a function of the time with the results of the present theory. In Figs. 2 and 3 we compare the "normalized" dimensionless function $K(x)$ as a function of the dimensionless variable $x$. Figure 2 is for the cases with $n=72$ while Fig. 3 is for $n=144$. According to the theory all the curves in Fig. 2 and 3 should overlap. In fact they agree very well for small times until $x \sim 4$ during which period the memory function falls by a factor of about 100 . According to our interpretation this is the time of about four times the duration of the binary collision and probably beyond the radius of convergence of a Taylor expansion, so that microscopically it is reasonably long. In Fig. 2 the curves from the theory and from the simulation B overlap and one cannot discriminate between them. After the "time" $x \approx 4$ the curves drift apart; the memory function derived from simulation eventually becomes negative, whereas, as pointed out in Sec. II the theoretical result remains positive for all times. At present we do not know whether this difference is due to shortcomings of the theory or limitations of the derivation from simulation or perhaps both.

\section{CONCLUSION}

As we pointed out in the Introduction, Dufty and Ernst [3], apparently starting from the same physical assumptions,

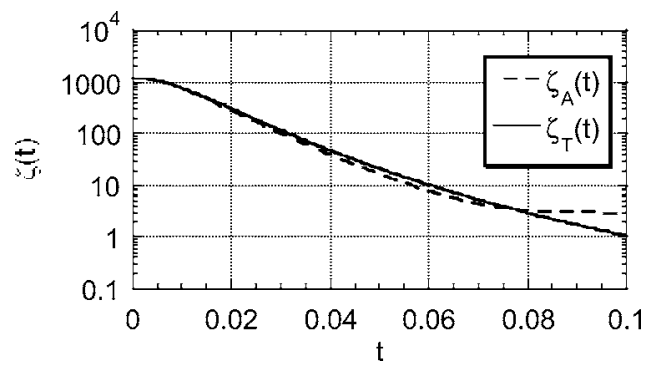

FIG. 1. A plot of the memory function, $\zeta(t)$, as a function of time. $\zeta(t)$ is measured in units of $k_{B} T / \sigma^{2}$ and time is measured in

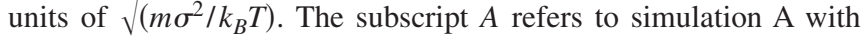
parameters as given in the table. The subscript $T$ refers to the result calculated from Eqs. (2.17)-(2.20).

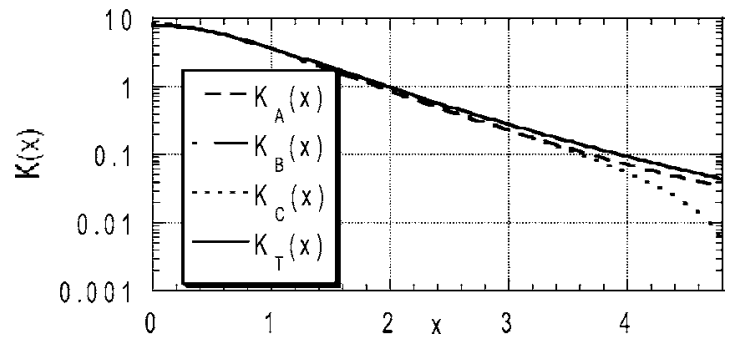

FIG. 2. A plot of the scaled function, $K(x)$, defined from the simulations by the method referred to in Sec. V and by Eqs. (2.20) for the theory. All these cases have $n=72$. The subscripts $A, B$, and $C$ refer to simulations $A, B$, and $C$, with parameters as given in the table. Both $K(x)$ and $x$ are dimensionless.

have derived scaling formulas for the VACF and FACF at short times in the hard sphere limit. There should be a relationship between their scaling functions and our scaling function, $K(x)$, but we have not yet established this. They claim that their results are asymptotically exact in the hard sphere limit of infinite $n$, and they certainly agree very well with data for Molecular Dynamics (MD) simulations using $n=1152$ (see Ref. [3]). Our comparison with simulation suggests that for the memory function the validity extends down to at least $n \approx 72$. Further work clearly needs to be carried out to establish the domain of applicability of these various approaches.

In summary, on the assumption that binary collisions are the dominant scattering process, we have derived a closed explicit and tractable formula for the velocity autocorrelation function memory function for a fluid of molecules interacting through a steeply repulsive potential. This result reduces to that of Enskog in the limit of hard spheres and is a generalization of his result. It has been shown that the final expression agrees with that derived from Molecular Dynamics simulation of steeply repulsive particles for short times. Systems with interaction potentials of the kind studied here are more relevant to colloidal systems. Although such potentials are not realistic for simple molecular liquids, there is a dearth of potential forms for which accurate results can be derived. In the absence of more realistic systems Enskog's theory has proved to be a useful guide in the past. Because of the more realistic systems studied in this paper there is some expectation that the new results presented here are simple enough to prove helpful in future studies.

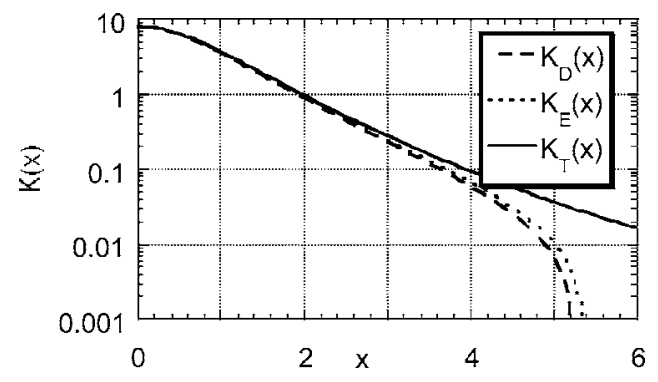

FIG. 3. The notation is the same as for Fig. 2. These cases have $n=144$. The subscripts $D$ and $E$ refer to simulations $D$ and $E$, with parameters as given in the table. 


\section{APPENDIX}

Here we outline the solution of the equations of motion. In this analysis $\mathbf{r}, \mathbf{p}$ are the initial values of the relative positions and momenta of the two molecules and we denote by $R$ the separation $r(t)$ at the later time $t$. We require only the conservation equations for the angular momentum and the energy. Together they yield the single equation for $R$,

$$
\frac{m}{4}\left(\dot{R}^{2}+\frac{4 l^{2}}{m^{2} R^{2}}\right)+\phi(R)=E \equiv \frac{p^{2}}{m}+\phi(r) .
$$

In this equation $l$ is the conserved angular momentum given by

$$
l=p R \sin \theta .
$$

To solve Eq. (A1) for $R(t)$ we exploit the fact that the potential is steeply rising and repulsive. In that case according to our previous work [11] the gradient of the pair distribution function which appears in the integrand of Eq. (2.2) has a narrow peak when $r, R \sim \sigma$, the width of the peak being proportional to $\sigma / n$. Furthermore nearly all the scattering takes place within a region where $R \sim \sigma$, indeed $R-\sigma$ $\sim \sigma / n$. However, although $R$ varies little in the integrand the potential can and does vary between 0 and $\infty$. We exploit these features of the problem to solve Eq. (A1) analytically.

We begin by changing the dependent variable to $\phi$ and use the fact that

$$
\dot{\phi}=-n \varepsilon \frac{\dot{R}}{R}\left(\frac{\sigma}{R}\right)^{n}=-\frac{n}{R} \phi \dot{R}
$$

to substitute for $\dot{R}$ in Eq. (A1). At the same time we approximate by replacing $R$ by $\sigma$. The result is

$$
\left(\frac{\sigma \dot{\phi}}{n \phi}\right)^{2}=\frac{4 E}{m}-\frac{4 l^{2}}{m^{2} \sigma^{2}}-\frac{4 \phi}{m} \equiv \frac{4}{m}(W-\phi) .
$$

This equation can be integrated to relate the value of $\phi$ at time $t$, namely $\phi(R)$, to that at the initial time, $\phi(r)$, and, after some reordering, yields

$$
\frac{1-\sqrt{1-\phi(R) / W}}{1+\sqrt{1-\phi(R) / W}}=\frac{1-\sqrt{1-\phi(r) / W}}{1+\sqrt{1-\phi(r) / W}} \exp ( \pm \gamma t),
$$

where $\gamma=2 n \sqrt{W / m \sigma^{2}}$.

The sign of the exponent depends on whether the molecules are initially approaching each other or not. Note that when the sign is negative, $\phi(R)$ decreases to zero with time and the molecules are separating. Similarly when the sign is positive $\phi(R)$ increases with time and the molecules are approaching. Initially the relative radial momentum of the molecules is

$$
\mathbf{p} \cdot \mathbf{r} / r=p \cos \theta \equiv p \mu,
$$

and if this is positive the molecules are separating initially and they continue to separate thereafter. Some straightforward but tedious manipulation shows that in all cases the result can be written

$$
\frac{\phi(R)}{W}=\frac{4 \exp \left\{-\gamma\left[t+t_{m} \operatorname{sgn}(\cos \theta)\right]\right\}}{\left(1+\exp \left\{-\gamma\left[t+t_{m} \operatorname{sgn}(\cos \theta)\right]\right\}\right)^{2}},
$$

where $t_{m}$ is the time when the molecules are at their closest distance of approach. It is given by

$$
\exp \left(-\gamma t_{m}\right)=\frac{\sqrt{W}-\sqrt{W-\phi(r)}}{\sqrt{W}+\sqrt{W-\phi(r)}} .
$$

The sign in Eq. (A6) takes care of the fact that if the molecules are separating initially their closest distance of approach was in the past (before $t=0$ ).
[1] P. Résibois and M. de Leener, Classical Kinetic Theory of Fluids (Wiley, New York, 1977).

[2] H. Sigurgeirsson and D. M. Heyes, Mol. Phys. 101, 469 (2003).

[3] J. W. Dufty and M. H. Ernst, Mol. Phys. 102, 2123 (2004).

[4] P. Boon and S. Yip, Molecular Hydrodynamics (McGraw-Hill, New York, 1980).

[5] M. S. Green, J. Chem. Phys. 22, 398 (1954).

[6] R. Kubo, J. Phys. Soc. Jpn. 12, 570 (1957).

[7] J. P. Hansen and I. R. McDonald, The Theory of Simple Liquids (Academic Press, London, 1986).

[8] L. L. Lee and T.-H. Chung, J. Chem. Phys. 77, 4650 (1982).

[9] R. K. Sharma, R. K. Moudgil, and K. Tankeshwar, Phys. Rev. E 54, 3652 (1996).

[10] K. Miyazaki, G. Srinivas, and B. Bagchi, J. Chem. Phys. 114, 6276 (2001). This paper is referred to as MSB in this paper.

[11] D. M. Heyes, J. G. Powles, and G. Rickayzen, Mol. Phys. 100, 595 (2002); D. M. Heyes, G. Rickayzen, and A. C. Brańka, ibid. 102, 2057 (2004).

[12] J.-P. Hansen and I. R. McDonald, Theory of Simple Liquids, 2nd ed. (Academic Press, London, 1986), Sec. 7.2, pp. 202-
203 [but note there is a misprint in Eq. (7.2.19) where $3 / 2$ should be replaced by 2/3]; P. Boon and S. Yip, Theory of Simple Liquids, 2nd ed., p. 147.

[13] R. Zwanzig, in Lectures in Theoretical Physics, edited by W. E. Britton, B. W. Downs, and J. Downs (Wiley Interscience, New York, 1961), Vol. III, p. 135.

[14] H. Mori, Prog. Theor. Phys. 33, 423 (1965); 34, 399 (1965).

[15] G. F. Mazenko and S. Yip, in Modern Theoretical Chemistry, edited by B. J. Berne (Plenum, New York, 1977), Vol. 6, p. 181.

[16] J.-P. Hansen and I. R. McDonald, in Modern Theoretical Chemistry (Ref. [12]), p. 211.

[17] J. A. Barker and D. Henderson, J. Chem. Phys. 47, 4714 (1967).

[18] G. Rickayzen, J. G. Powles, and D. M. Heyes, J. Chem. Phys. 118, 11048 (2003).

[19] J.-P. Hansen and I. R. McDonald, in Modern Theoretical Chemistry (Ref. [12]), p. 204.

[20] N. F. Carnahan and K. E. Starling, J. Chem. Phys. 51, 635 (1969). 